\title{
Qualidade de vida e independência funcional de idosos frequentadores do clube do idoso do município de Sorocaba
}

\author{
Quality of life and functional independence of the elderly \\ goers to the elderly house in the city of Sorocaba
}

Bruna Malavazzi Lima1 ${ }^{1}$, Franciele Andrade Araujo¹, Fátima Ayres de Araújo Scattolin ${ }^{1}$

${ }^{1}$ Curso de Enfermagem, Faculdade de Ciências Médicas e da Saúde (FCMS), Pontifícia Universidade Católica de São Paulo (PUC-SP) - São Paulo (SP), Brasil.

DOI: http://dx.doi.org/10.7322/abcshs.v41i3.907

\begin{abstract}
RESUMO
Introdução: Nos últimos anos a expectativa de vida vem aumentando em todo o mundo. A manutenção da capacidade funcional e da qualidade de vida (QV) dessa população é um dos desafios da atualidade. Objetivo: Avaliar a QV e a independência funcional de idosos frequentadores do Clube do Idoso do município de Sorocaba e investigar a correlação entre essas medidas. Métodos: Participaram idosos de ambos os sexos com idade $\geq 60$ anos. Foram utilizados: Instrwumento de Caracterização Sociodemográfica; World Health Organization Quality of Life Assessment-Old (WHOQOL-OLD) e Medida de Independência Funcional (MIF). Análises estatísticas realizadas: teste de Mann-Whitney e teste de Kruskal-Wallis para comparação entre os escores e o coeficiente de correlação de postos de Spearman para verificar a correlação entre os instrumentos. Resultados: Participaram 71 idosos, 69,01\% mulheres, 56,34\% com idade entre 60 e 69 anos, 33,80\% casados e $83,10 \%$ aposentados. Não houve diferenças significativas quanto ao sexo para os dois instrumentos e quanto à idade nos escores do WHOQOL-OLD. Os idosos $\geq 80$ anos apresentaram queda da independência funcional $(p<0,001)$ e aqueles com mais anos de estudo apresentaram maior independência funcional e avaliaram melhor a QV global. Houve correlação diretamente proporcional entre escores da MIF e QV. Conclusão: Este estudo apontou que a funcionalidade está diretamente ligada à QV. Daí a importância dos grupos de terceira idade como forma de favorecer a prática de atividade física e aumentar a interação social, fatores essenciais na proteção e promoção da saúde e da QV dessa população.
\end{abstract}

Palavras-chave: idoso; qualidade de vida; saúde do idoso; envelhecimento.

\begin{abstract}
Introduction: In the last years, life expectancy has been increasing all over the world. Preserving the functional capacity and the quality of life $(\mathrm{QOL})$ of this population is one of the current challenges. Objective: Assess the $\mathrm{QOL}$ and the functional independence of the elderly attending the Elderly Club in the Municipality of Sorocaba and to investigate the correlation among these measures. Methods: Elderly men and women aged $\geq 60$ years old participated in the assessment. The instruments used were Sociodemographic Characterization Instrument; World Health Organization Quality of Life Assessment - Old (WHOQOL-OLD) and Functional Independence Measure (FIM). Statistical analysis: Mann-Whitney and Kruskal-Wallis tests to compare the scores and Correlation of Spearman to check the correlation between instruments. Results: Seventy-one elderly participated, being $69.01 \%$ women, $56.34 \%$ between 60 and 69 years old, 33.80\% married and $83.10 \%$ retired. There were not significant differences regarding sex between the two instruments and, in the scores of WHOQOL-OLD, regarding age. The elderly aged $\geq 80$ years old showed a decline in the functional independence $(p<0.001)$ and those with more years of study showed a better functional independence and assessed better the global QOL. There was a directly proportional correlation between the scores of the MIF and the QOL. Conclusion: This study indicated that the functionality is directly linked to QOL. Hence the importance of the elderly groups as a way of encouraging the practice of physical activities and increasing social interaction, essential factors for the protection and promotion of health and QOL of this population.
\end{abstract}

Keywords: aged; quality of life; health of the elderly; aging

Recebido em: 03/12/2015

Revisado em: 06/05/2016

Aprovado em: 30/06/2016

Autor para correspondência: Bruna Malavazzi Lima - Faculdade de Medicina, Pontifícia Universidade Católica de São Paulo, Campus Sorocaba Rua Joubert Wey, 290 - Jardim Vergueiro - CEP: 18030-070 - Sorocaba (SP), Brasil - E-mail: brumalavazzi@hotmail.com Conflito de interesses: nada a declarar. 


\section{INTRODUÇÃO}

A transição demográfica em todo o mundo revelou um aumento da população idosa. A população idosa brasileira, na primeira metade deste século, deverá crescer entre 2 e $4 \%$ ao ano, enquanto a população jovem tenderá a diminuir. De acordo com estimativas da Organização das Nações Unidas (ONU), a população idosa brasileira, que representava $3,1 \%$ da população total na década de 1970, deverá passar a aproximadamente 19\% em $2050^{1}$.

Aliado às condições da senescência, muitos idosos são submetidos a situações de isolamento social e dependência financeira, o que faz da velhice uma época de perdas, incapacidades e decrepitude, muito distante do conceito de envelhecimento bem-sucedido ${ }^{2}$.

Atualmente essa situação vem se acentuando, principalmente devido às mudanças na dinâmica familiar e às exigências do mundo moderno. A mulher deixou de ser a cuidadora e adentrou o mercado de trabalho, tanto como forma de realização pessoal como para colaborar nas despesas da casa ${ }^{3,4}$.

Essa mudança no perfil demográfico trouxe novos desafios às famílias, à sociedade e aos serviços de saúde. Novos espaços devem ser inaugurados para receber essa população que envelhece, nos quais o idoso possa se sentir acolhido em suas necessidades, fazer aquilo que gosta e, principalmente, se manter ativo.

Essa ideia vem se reproduzindo nos Centros de Convivência para Idosos como forma de atender a um dos objetivos norteadores da Política Nacional do Idoso (PNI), ou seja, a manutenção da capacidade funcional pelo maior tempo possível, favorecendo a independência e a autonomia da pessoa que envelhece.

O conceito de envelhecimento ativo pressupõe a independência funcional como seu principal marcador de saúde. A capacidade funcional surge como um novo parâmetro de saúde ${ }^{5}$ e está diretamente ligada à qualidade de vida $(\mathrm{QV})$.

No sentido de entender a QV como um conceito amplo que engloba a saúde física e o nível de independência, as relações sociais, o estado psicológico, as crenças e o meio ambiente, a Organização Mundial da Saúde (OMS) definiu QV da seguinte forma: "a percepção do indivíduo de sua posição na vida, no contexto de sua cultura e dos sistemas de valores em que vive e em relação a suas expectativas, seus padrões e suas preocupações”. Essa definição orientou o projeto de desenvolvimento de um instrumento para medir esse construto ${ }^{6}$.

A manutenção da funcionalidade do indivíduo que envelhece é um grande desafio. Daí a importância de instrumentos que identifiquem as perdas funcionais e cognitivas no dia a dia que atuam como fatores de risco para o comprometimento da capacidade funcional ${ }^{7,8}$. Um dos instrumentos mais utilizados para a avaliação da funcionalidade é a Medida de Independência Funcional (MIF), uma escala específica que avalia o desempenho do indivíduo nas atividades de vida diária (AVDs) ${ }^{9-11}$.

Considerando o aumento da população idosa e a importância da manutenção da capacidade funcional pelo maior tempo possível como condição favorável para um envelhecimento bem-sucedido, este estudo teve como objetivos: avaliar a QV e a independência funcional dos idosos frequentadores do Clube do Idoso do município de Sorocaba, assim como investigar a relação entre a QV e a funcionalidade desses mesmos idosos.

\section{MÉTODOS}

Trata se de um estudo de natureza exploratória, quantitativo, correlacional, que foi conduzido no Clube do Idoso "Carlos Alberto Moura Pereira da Silva”, no município de Sorocaba. A instituição é coordenada pela Secretaria do Desenvolvimento Social e tem como objetivo oferecer mais QV aos idosos por meio de atividades recreativas que estimulem a socialização e a autoestima dos frequentadores. O Clube funciona de segunda a sexta-feira, das 8 às 17 horas, e oferece aos participantes a oportunidade de realizar atividade física (aulas de tai chi chuan, ginástica e alongamento), dança circular e de ritmos e dança de salão, cursos de teatro e de fotografia, pintura em tecido e em tela, palestras educativas e oficinas.

Fizeram parte do estudo sujeitos de ambos os sexos, com idade igual ou superior a 60 anos. Compuseram a amostra os idosos que concordaram em participar de livre vontade e assinaram o Termo de Consentimento Livre e Esclarecido (TCLE). Aqueles idosos que apresentaram limitações que impossibilitavam a comunicação e a expressão e aqueles que se recusaram a participar foram excluídos do estudo.

O tamanho da amostra foi calculado com base na análise multivariada para estudar a relação das variáveis de interesse com os escores de QV e de independência funcional, também considerando os coeficientes de correlação de Spearman entre os escores (total e por domínios) dos instrumentos: World Health Organization Quality of Life Aassessment-Old (WHOQOL-OLD) e MIF obtidos nas análises do estudo piloto $(n=25)$, estimando coeficiente de correlação em torno de 0,30 , com nível de significância $(\alpha)$ de $5 \%$ e poder do teste de $80 \%(\beta)$ de $20 \%{ }^{12,13}$.

Dessa forma, foi obtida uma amostra total de 80 sujeitos. No entanto, no prazo estabelecido para a coleta de dados (fevereiro a abril de 2015), o número de idosos que estavam frequentando o Clube foi menor que o esperado, resultando em uma amostra de 71 sujeitos.

As visitas para a realização das entrevistas foram feitas durante a semana em períodos diferentes para garantir a participação do maior número de idosos. A coleta de dados foi iniciada após os esclarecimentos sobre os objetivos da pesquisa e o consentimento dos sujeitos em participar por meio da assinatura do TCLE.

Os instrumentos utilizados para a coleta de dados foram os seguintes:

- Instrumento de Caracterização dos Sujeitos: foi construído um instrumento com os seguintes itens: nome, idade, estado civil, sexo, composição familiar, procedência, escolaridade e religião, hábitos e estilos de vida: lazer, atividade física, tabagismo e consumo de álcool e morbidades referidas. 
- WHOQOL-OLD: trata-se de um módulo específico de avaliação da QV em idosos com propriedades de uso transcultural $^{14}$. O desenvolvimento desse instrumento foi conduzido pelo Grupo WHOQOL em um projeto colaborativo internacional, seguindo as mesmas diretrizes do WHOQOL-100 e do WHOQOL-BREF. O centro brasileiro do Grupo WHOQOL participou do projeto, dando origem à versão brasileira, cujas propriedades psicométricas foram avaliadas por Fleck et al..$^{15}$ em 2006. O instrumento é composto por 24 itens divididos em 6 domínios. Cada domínio possui 4 itens, que geram escores que variam de 4 a 20 pontos. Os escores dos 6 domínios, combinados com as respostas aos 24 itens, geram também um escore total (que varia de 24 a 120). O WHOQOL-OLD possui seis domínios. São eles:

1. Funcionamento dos sentidos (FS): avalia o impacto da perda do funcionamento dos sentidos (audição, visão, paladar, olfato e tato) na QV.

2. Autonomia (AU): avalia a independência do idoso para tomar as suas próprias decisões, sentir que controla o seu futuro, fazer as coisas que gostaria de fazer e acreditar que as pessoas ao seu redor respeitam a sua liberdade.

3. Atividades passadas, presentes e futuras (PPF): reflete a satisfação com as realizações na vida, as oportunidades de novas relações, o reconhecimento merecido na vida, a felicidade com que espera o futuro e os objetivos a serem alcançados.

4. Participação social (PS): demonstra a satisfação social com as atividades diárias, com o uso do tempo, com o nível pessoal de atividades e com as oportunidades de participar nas atividades da comunidade.

5. Morte e morrer (MEM): avalia as percepções e medos acerca da morte e do morrer.

6. Intimidade (INT): avalia a capacidade de estabelecer relacionamentos íntimos e pessoais.

Os escores mais altos indicam melhor QV associada aos domínios e os valores mais baixos, pior QV.

A pontuação dos itens do questionário obedece a critérios definidos:

- Para itens expressos positivamente, os valores mais elevados representam melhor QV.

- Para itens expressos negativamente, o escore deve ser recodificado (itens 1, 2, 6, 7, 8, 9 e 10) de forma que os valores numéricos atribuídos sejam invertidos $(1=5,2=4,3=3,4=2$ e $5=1)$.

- A soma dos itens que pertencem a um domínio produz o escore bruto daquele domínio.

- A transformação de um escore bruto em um escore transformado da escala possibilita expressar o escore em porcentagem seguindo a regra de transformação: $\mathrm{ET}=6,25 \mathrm{x}(\mathrm{EB}-4)$, na qual $\mathrm{ET}=$ escore transformado e $\mathrm{EB}=$ escore bruto.

- Os escores dos 6 domínios ou os valores dos 24 itens do módulo WHOQOL-OLD combinados podem produzir um escore geral para a QV (QV global).
- $\quad$ MIF: trata-se de um instrumento específico para a avaliação da funcionalidade por meio do desempenho do indivíduo nas AVDs. A MIF foi desenvolvida pela Academia Americana de Reabilitação na década de 1980 com o objetivo de padronizar conceitos sobre incapacidades e obter um instrumento que avaliasse as AVDs e permitisse a avaliação globalizada da reabilitação. Foi publicada em $1986^{\circ}$. No Brasil, a versão foi traduzida e validada por Riberto et al. ${ }^{10}$, demonstrando propriedades psicométricas satisfatórias do instrumento. A MIF consiste em um conjunto de 18 tarefas que compõem duas subescalas: a motora (MIFm) e a cognitiva/social (MIFcs). A MIFm avalia o autocuidado, o controle esfincteriano, a mobilidade e a locomoção e a MIFcs avalia a comunicação e a cognição social. Cada item é classificado em uma escala de graus de dependência de sete níveis, sendo que 7 equivale à independência completa (normalidade na realização de tarefas) e 1, à dependência total. As somas das pontuações dos itens da MIF podem gerar um escore para cada subescala (MIFm e MIFcs) e um escore total obtido pela soma de todos os itens. A variação possível para o instrumento está entre 18 e 126 , sendo que maiores escores revelam maior independência funcional.

Para a aplicação do instrumento, foi realizado um treinamento sob orientação de uma das pesquisadoras, certificada pela Divisão de Medicina de Reabilitação - Hospital das Clínicas/Faculdade de Medicina da Universidade de São Paulo no Curso de capacitação para uso da MIF.

Foram realizadas as seguintes análises estatísticas: descritiva: com confecção de quadros de frequência, medidas de posição (média, mediana, valores mínimos e máximos) e dispersão (desvio padrão) para dados do instrumento de caracterização sociodemográfica, para os domínios e escore total do WHOQOL-OLD e da MIF; teste de Normalidade (ShapiroWilk e de Kolmogorov-Smirnov), que apontou que a maioria das variáveis teve desvio significativo de uma distribuição normal, determinando a utilização de testes não paramétricos; coeficiente alfa de Cronbach para verificar a consistência interna dos instrumentos WHOQOL-OLD e MIF; testes para comparação entre os escores do WHOQOL-OLD e da MIF e para as variáveis sociodemográficas (teste de Mann-Whitney para comparação entre dois grupos e teste de Kruskal-Wallis para comparação entre três ou mais grupos) e correlação de Spearman para verificar a correlação entre os domínios do WHOQOL-OLD e as subescalas da MIF.

Foi adotado o seguinte critério de classificação dos coeficientes de correlação: $<0,3$ (correlação de fraca magnitude), $\geq 0,3$ a $<0,5$ (correlação de moderada magnitude) e $\geq 0,5$ (correlação de forte magnitude), como preconizado por Ajzen e Fishbeim ${ }^{16}$, e utilizado um nível de significância de $5 \%$ (valor $\mathrm{p}<0,05$ ). A direção dessas correlações está relacionada à ordenação dos escores dos dois instrumentos, ou seja, quanto maiores os escores do 
WHOQOL-OLD, melhor a QV; e quanto mais elevados os escores da MIF, maior a independência funcional.

A pesquisa foi aprovada pelo Comitê de Ética em Pesquisa da Faculdade de Ciências Médicas e da Saúde da Pontifícia Universidade Católica de São Paulo (Parecer nº 95.0644 - CAAE 40113614.3.0000.5373).

\section{RESULTADOS}

Dos 71 idosos incluídos no estudo, 69,01\% (49/71) eram mulheres, 56,34\% tinham idade entre 60 a 69 anos, 33,80\% (24/71) eram casados, 83,10\% (59/71), aposentados e 53,52\% (38/71) tinham renda familiar média de 2 salários mínimos. As demais características sociodemográficas estão apresentadas na Tabela 1.

Em relação aos hábitos e estilos de vida, 71,83\% moravam com a família e apresentavam hábitos saudáveis, $91,55 \%$ não fumavam, $83,10 \%$ não faziam uso de bebida alcoólica e 90,14\% praticavam atividade física.

Das morbidades referidas pelos participantes da pesquisa, a hipertensão arterial sistêmica (HAS) (42,25\%), o diabetes mellitus (DM) $(22,53 \%)$ e as doenças osteoarticulares $(22,53 \%)$ apareceram em destaque.

Para a verificação da confiabilidade dos dois instrumentos foi aplicado o coeficiente de consistência interna (alfa

Tabela 1: Caracterização sociodemográfica dos 71 idosos frequentadores do Clube do Idoso de Sorocaba. Sorocaba. Sorocaba, São Paulo, 2015

\begin{tabular}{|c|c|c|}
\hline Variáveis & $\mathbf{n}$ & $\%$ \\
\hline Idade & 71 & \\
\hline 60-69 & 40 & 56,34 \\
\hline $70-79$ & 25 & 35,21 \\
\hline$\geq 80$ & 6 & 8,45 \\
\hline \multicolumn{3}{|l|}{ Sexo } \\
\hline Feminino & 49 & 69,01 \\
\hline Masculino & 22 & 30,99 \\
\hline \multicolumn{3}{|l|}{ Estado civil } \\
\hline Casado & 24 & 33,80 \\
\hline Viúvo & 22 & 30,99 \\
\hline Solteiro & 9 & 12,68 \\
\hline Outros & 16 & 22,54 \\
\hline \multicolumn{3}{|l|}{ Religião } \\
\hline Católica & 50 & 70,42 \\
\hline Evangélica & 10 & 14,08 \\
\hline Outras & 8 & 11,27 \\
\hline Não tem & 3 & 4,23 \\
\hline Renda* & 71 & \\
\hline \multicolumn{3}{|l|}{ Vínculo Empregatício } \\
\hline Do lar & 6 & 8,45 \\
\hline Pensionista & 6 & 8,45 \\
\hline Aposentado & 59 & 83,10 \\
\hline № de filhos & 71 & \\
\hline $\begin{array}{l}\text { Escolaridade } \\
\text { (anos de estudo) }\end{array}$ & 71 & \\
\hline Tempo que frequenta o clube ${ }^{\star *}$ & 71 & \\
\hline
\end{tabular}

*em salários mínimos; **em meses. de Cronbach), e verificou-se alta consistência interna para a maioria dos domínios de ambos os instrumentos (entre 0,62 e 0,85$)$. O domínio "Morte e morrer" teve o coeficiente mais baixo $(0,62)$.

A Tabela 2 apresenta os escores do WHOQOL-OLD global e por domínios obtidos junto aos 71 idosos estudados.

O domínio "Intimidade" foi o que obteve menor média, indicando que a QV está mais afetada nesse domínio em relação aos demais. O que se observa também é que os escores de todos os domínios e da QV global do WHOQOL-OLD estão elevados, se considerarmos os valores mínimo e máximo e as medianas para cada domínio e para a QV global.

A média dos escores obtidos para a MIFm foi de 88,72 ( $\pm 6,09)$ e para a MIFcs, de 32,73 $( \pm 2,84)$. Considerando a amplitude de cada subescala, observa-se que esses valores estão muito próximos do valor máximo, o que sugere que os sujeitos da pesquisa estão independentes para as AVDs. Na Tabela 3 estão apresentados os escores da MIF total (MIFt), da MIFm e da MIFcs.

Na comparação entre homens e mulheres não houve diferenças significativas para o WHOQOL-OLD e para a MIF.

Considerando-se as faixas etárias, as diferenças entre as médias dos escores do WHOQOL-OLD, tanto para os domínios como para a QV global, também não foram significativas. Ao contrário, para a MIF total e as subescalas, as diferenças entre as médias dos escores foram significativas, ou seja, a faixa dos idosos $\geq 80$ anos tem menor independência funcional (Tabela 4).

Tabela 2: Escores transformados do World Health Organization Quality of Life Assessment-Old por domínios e global dos 71 idosos frequentadores do Clube do Idoso de Sorocaba. Sorocaba, São Paulo, 2015

\begin{tabular}{|l|c|c|c|c|c|}
\hline $\begin{array}{l}\text { Domínios do } \\
\text { WHOQOL-OLD }\end{array}$ & Média & DP & Mínimo & Mediana & Máximo \\
\hline $\begin{array}{l}\text { Funcionamento } \\
\text { dos sentidos }\end{array}$ & 84,33 & 16,73 & 25,00 & 93,75 & 100,00 \\
\hline $\begin{array}{l}\text { Autonomia } \\
\text { Atividades }\end{array}$ & 74,30 & 14,47 & 31,25 & 75,00 & 100,00 \\
\hline $\begin{array}{l}\text { passadas, } \\
\text { presentes e futuras }\end{array}$ & 71,21 & 11,55 & 50,00 & 68,75 & 100,00 \\
\hline Participação social & 75,97 & 8,09 & 56,25 & 75,00 & 100,00 \\
\hline Morte e morrer & 76,50 & 18,97 & 25,00 & 81,25 & 100,00 \\
\hline Intimidade & 69,81 & 16,87 & 25,00 & 75,00 & 100,00 \\
\hline $\begin{array}{l}\text { Qualidade de vida } \\
\text { global }\end{array}$ & 75,35 & 8,66 & 51,04 & 75,00 & 93,75 \\
\hline
\end{tabular}

WHOQOL-OLD: World Health Organization Quality of Life Assessment-Old.

Tabela 3: Escores da Medida de Independência Funcional, subescalas e total dos 71 idosos pesquisados. Sorocaba, São Paulo, 2015

\begin{tabular}{|l|c|c|c|c|c|c|c|}
$\begin{array}{l}\text { MIF e } \\
\text { subescalas }\end{array}$ & $\mathbf{n}$ & Média & DP & Mínimo & Mediana & Máximo & $\begin{array}{l}\text { Variação } \\
\text { possível }\end{array}$ \\
\hline MIFm & 71 & 88,72 & 6,09 & 43,00 & 90,00 & 91,00 & $13-91$ \\
\hline MIFc/s & 71 & 32,73 & 2,84 & 18,00 & 34,00 & 35,00 & $5-35$ \\
\hline MIFt & 71 & 121,5 & 8,45 & 63,00 & 124,0 & 126,0 & $18-126$ \\
\hline
\end{tabular}

MIF: Medida de Independência Funcional; DP: desvio padrão; MIFm: Medida de Independência Funcional motora; MIFc/s: Medida de Independência Funcional cognitiva/social; MIFt: Medida de Independência Funcional total. 
Quando observada a variável "Escolaridade", as diferenças nas médias dos escores foram significativas para a QV global e para as subescalas e MIF total, indicando que aqueles idosos com mais anos de estudo tinham maior independência funcional e avaliaram melhor a QV global (Tabela 5).

A Tabela 6 aponta a correlação entre os escores do WHOQOOLD e da MIF. Para tanto, foi aplicado o coeficiente de correlação de Spearman. O sentido direto das correlações entre as variáveis

Tabela 4: Comparação entre as médias dos escores do World Health Organization Quality of Life Assessment-Old e da Medida de Independência Funcional de acordo com as faixas etárias dos 71 idosos frequentadores do Clube do Idoso de Sorocaba. Sorocaba, São Paulo, 2015

\begin{tabular}{|l|c|c|c|c|}
\multirow{2}{*}{ WHOQOL-OLD/MIF } & \multicolumn{3}{|c|}{ Idade (anos) } \\
\cline { 1 - 4 } & $\begin{array}{c}\mathbf{6 0 - 6 9} \\
\mathbf{n}=\mathbf{4 0}\end{array}$ & $\begin{array}{c}\mathbf{7 0 - 7 9} \\
\mathbf{n}=\mathbf{2 5}\end{array}$ & $\begin{array}{c}\mathbf{2 8 0} \\
\mathbf{n}=\mathbf{2 5}\end{array}$ & Valor $\mathbf{p}$ \\
\hline $\begin{array}{l}\text { Funcionamento } \\
\text { dos sentidos }\end{array}$ & 85,0 & 87,00 & 68,75 & 0,148 \\
\hline Autonomia & 75,16 & 75,25 & 64,58 & 0,361 \\
\hline $\begin{array}{l}\text { Atividades passadas, } \\
\text { presentes e futuras }\end{array}$ & 71,09 & 72,25 & 67,71 & 0,641 \\
\hline Participação social & 75,47 & 77,25 & 73,96 & 0,627 \\
\hline Morte e morrer & 76,09 & 75,50 & 83,33 & 0,804 \\
\hline Intimidade & 72,19 & 69,00 & 57,29 & 0,080 \\
\hline $\begin{array}{l}\text { Qualidade de vida } \\
\text { global }\end{array}$ & 75,83 & 76,04 & 69,27 & 0,160 \\
\hline MIFm & 90,03 & 88,88 & 79,33 & $p<0,001$ \\
\hline MIFc/s & 33,38 & 32,72 & 28,50 & $p<0,001$ \\
\hline MIFt & 123,40 & 121,60 & 107,83 & $p<0,001$ \\
\hline
\end{tabular}

WHOQOL-OLD: World Health Organization Quality of Life Assessment-Old MIF: Medida de Independência Funcional; MIFm: Medida de Independência Funcional motora; MIFc/s: Medida de Independência Funcional cognitiva/social; MIFt: Medida de Independência Funcional total. Diferenças significativas/teste de comparações múltiplas de Dunn $(p<0,05)$.

Tabela 5: Comparação entre as médias dos escores do World Health Organization Quality of Life Assessment-Old e da Medida de Independência Funcional de acordo com a escolaridade dos 71 idosos frequentadores do Clube do Idoso de Sorocaba. Sorocaba São Paulo, 2015

\begin{tabular}{|c|c|c|c|c|}
\hline \multirow{2}{*}{ WHOQOL-OLD/MIF } & \multicolumn{3}{|c|}{$\begin{array}{c}\text { Escolaridade } \\
\text { (anos de estudo) }\end{array}$} & \multirow{2}{*}{ Valor $p$} \\
\hline & $\begin{array}{c}0-4 \\
n=16\end{array}$ & $\begin{array}{c}5-8 \\
n=16\end{array}$ & $\begin{array}{c}\geq 9 \\
\mathrm{n}=32\end{array}$ & \\
\hline $\begin{array}{l}\text { Funcionamento dos } \\
\text { sentidos }\end{array}$ & 79,62 & 85,16 & 87,31 & 0,051 \\
\hline Autonomia & 73,37 & 70,31 & 76,95 & 0,309 \\
\hline $\begin{array}{l}\text { Atividades passadas, } \\
\text { presentes e futuras }\end{array}$ & 69,57 & 69,53 & 73,24 & 0,379 \\
\hline Participação social & 74,46 & 76,17 & 76,95 & 0,192 \\
\hline Morte e morrer & 68,21 & 78,13 & 81,64 & 0,06 \\
\hline Intimidade & 63,86 & 69,53 & 74,22 & 0,07 \\
\hline $\begin{array}{l}\text { Qualidade de vida } \\
\text { global }\end{array}$ & 71,51 & 74,80 & 78,39 & 0,015 \\
\hline MIFm & 88,70 & 89,06 & 88,56 & 0,018 \\
\hline $\mathrm{MIFc} / \mathrm{s}$ & 31,52 & 33,25 & 33,44 & $<0,001$ \\
\hline $\mathrm{MIFt}$ & 120,22 & 122,50 & 121,81 & $<0,001$ \\
\hline
\end{tabular}

WHOQOL-OLD: World Health Organization Quality of Life Assessment-Old; MIF Medida de Independência Funcional; MIFm: Medida de Independência Funciona motora; MIFc/s: Medida de Independência Funcional cognitiva/social; MIFt: Medida de Independência Funcional total. confirma que ambos os instrumentos são diretamente correlacionados (maiores valores do WHOQOL-OLD=melhor QV; maiores valores da $\mathrm{MIF}=$ maior independência funcional). Quanto à força das correlações, foram encontradas correlações significativas de moderada magnitude, sendo o maior valor de correlação observado entre os domínios "Autonomia e MIFm" $(0,44)$ e "QV global e MIFm" $(0,44)$.

\section{DISCUSSÃO}

O perfil sociodemográfico da amostra retrata uma população idosa, composta em sua maioria por mulheres $(69,01 \%)$ com idade variando entre 60 e 69 anos. O destaque para a população feminina pode estar relacionado à maior longevidade das mulheres, o que caracteriza a feminização do envelhecimento. Outros estudos encontraram resultados semelhantes e atribuíram às mulheres atitudes mais positivas em relação à saúde, com menor exposição aos fatores de risco do que os homens ${ }^{17-19}$.

Foi observada uma média de 17 anos de estudo entre os entrevistados, o que parece ter tido influência importante na QV dessas pessoas, inclusive na busca por participação em grupos e troca de experiências. A amostra deste estudo foi composta de uma parcela diferenciada dessa população, uma vez que, segundo dados do Instituto Brasileiro de Geografia e Estatística (IBGE), 24\% da população brasileira com mais de 60 anos é analfabeta ${ }^{20}$.

Em relação ao estado conjugal, a maior parte dos entrevistados era casada e viúva, vivendo da aposentadoria, com renda média de dois salários mínimos. Essa também é uma realidade da população idosa brasileira. Um pequeno contingente de idosos tem condições materiais e acesso aos meios para viver com saúde e QV.

Tabela 6: Valores do coeficiente de orrelação de Spearman entre os escores do WHOQOL-OLD: World Health Organization Quality of Life Assessment-Old (domínios e qualidade de vida global) e Medida de Independência Funcional (total e subescalas). Sorocaba, São Paulo, 2015

\begin{tabular}{|c|c|c|c|}
\hline \multirow{2}{*}{ WHOQOL/Domínios } & \multicolumn{3}{|c|}{ MIF } \\
\hline & MIFm & MIFc/s & MIFt \\
\hline $\begin{array}{l}\text { Funcionamento } \\
\text { dos sentidos }\end{array}$ & $\begin{array}{c}r=0,41 \\
p=0,0003\end{array}$ & $\begin{array}{c}r=0,39 \\
p=0,007\end{array}$ & $\begin{array}{c}r=0,41 \\
p=0,004\end{array}$ \\
\hline Autonomia & $\begin{array}{c}r=0,44 \\
p=0,001\end{array}$ & $\begin{array}{c}r=0,33 \\
p=0,004\end{array}$ & $\begin{array}{c}r=0,41 \\
p=0,003\end{array}$ \\
\hline $\begin{array}{l}\text { Atividades passadas, } \\
\text { presentes e futuras }\end{array}$ & $\begin{array}{l}r=0,19 \\
p=0,10\end{array}$ & $\begin{array}{l}r=0,17 \\
p=0,14\end{array}$ & $\begin{array}{l}r=0,20 \\
p=0,08\end{array}$ \\
\hline Participação social & $\begin{array}{l}r=0,11 \\
p=0,32\end{array}$ & $\begin{array}{l}r=0,13 \\
p=0,24\end{array}$ & $\begin{array}{l}r=0,13 \\
p=0,24\end{array}$ \\
\hline Morte e morrer & $\begin{array}{l}r=0,04 \\
p=0,70\end{array}$ & $\begin{array}{l}r=0,02 \\
p=0,86\end{array}$ & $\begin{array}{l}r=0,05 \\
p=0,63\end{array}$ \\
\hline Intimidade & $\begin{array}{l}r=0,39 \\
p=0,008\end{array}$ & $\begin{array}{l}r=0,17 \\
p=0,15\end{array}$ & $\begin{array}{c}r=0,31 \\
p=0,007\end{array}$ \\
\hline Qualidade de vida global & $\begin{array}{c}r=0,44 \\
p=0,001\end{array}$ & $\begin{array}{c}r=0,33 \\
p=0,004\end{array}$ & $\begin{array}{c}r=0,42 \\
p=0,002\end{array}$ \\
\hline
\end{tabular}

WHOQOL: World Health Organization Quality of Life Assessment; MIF: Medida de Independência Funcional; DP: desvio padrão; MIFm: Medida de Independência Funcional motora; MIFc/s: Medida de Independência Funcional cognitiva/social; MIFt: Medida de Independência Funcional total.

${ }^{*}$ r: coeficiente de correlação de Spearman; $p$ : valor $p$ 
Outra parte dessa população vive com aposentadorias mínimas e, no dizer de um aposentado, "quase sempre a conta não fecha" ${ }^{21}$.

Nesta amostra, as morbidades referidas mais frequentemente foram: DM e HAS. No Projeto SABE, um inquérito sobre Saúde, Bem-Estar e Envelhecimento realizado no município de São Paulo, no capítulo sobre Condições de Saúde, aparecem também a HAS e o DM entre as cinco doenças crônicas mais referidas ${ }^{22}$. A HAS é o mais importante fator de risco cardiovascular modificável, estando associada a complicações bastante frequentes em idosos. Segundo o Departamento de Informática do Sistema Único de Saúde (DATASUS), 35,2\% da população com 60 anos ou mais vão a óbito por essa causa. Na análise da QV e adesão a tratamentos não farmacológicos para essas patologias (exercício físico; controle alimentar; redução de peso), percebe-se que esse grupo de idosos tem maior chance de sobrevida ${ }^{23}$.

Nessa direção, é importante considerar que a amostra deste estudo tem características importantes em relação aos hábitos e estilos de vida: são idosos que se mantêm ativos tanto em relação à parte física como cognitiva, o que tem um reflexo importante na QV dessas pessoas. Estudos mostram que idosos que estão inseridos na sociedade e participam de grupos de convivência fazem uma avaliação mais favorável da sua QV ${ }^{24,25}$.

Pela análise dos escores do WHOQOL-OLD e da MIF foi possível perceber que os idosos entrevistados podem ser considerados independentes para as AVDs e fizeram uma avaliação satisfatória da QV. O idoso que se mantém ativo tem maior satisfação com a vida e, consequentemente, melhor $\mathrm{QV}^{26}$. Isso é especialmente importante em espaços onde o idoso possa desenvolver relações sociais, ter vínculos de amizade e exercer sua cidadania. As atividades desenvolvidas no Clube do Idoso (ginástica, dança, jogos, teatro, grupos de artesanato) trazem benefícios que auxiliam o idoso a manter a independência e a autonomia. Esses idosos ampliam suas redes de apoio social e desenvolvem autoestima e autoconfiança. Do mesmo modo, a prática regular de atividade física aumenta a capacidade aeróbica, a flexibilidade, o equilíbrio, a resistência física e a força muscular, trazendo benefícios também para a cognição, a memória e as habilidades sociais ${ }^{27}$.

Neste estudo, nas faixas etárias consideradas (60-69; 70-79; $\geq 80$ anos), houve diferença significativa nos escores da MIF, indicando queda da independência funcional com o avançar da idade. O desempenho funcional, ou seja, a condição que o indivíduo possui de viver de maneira autônoma e se relacionar em seu meio diminui conforme a idade aumenta. O mesmo resultado foi demonstrado em outros estudos ${ }^{28,29}$.

Embora se encontre na literatura estudo mostrando que a idade avançada foi associada aos mais baixos escores de $\mathrm{QV}^{30}$, este estudo não evidenciou a influência da idade nos escores de QV, sugerindo que a idade avançada, por si só, parece não piorar a $\mathrm{QV}^{31}$.

Nesta pesquisa, os maiores escores de independência funcional e QV foram associados ao maior grau de escolaridade. Outros estudos apresentaram resultados semelhantes ${ }^{32,33}$. A literatura aponta que os idosos com maior nível de escolaridade (médio ou superior e fundamental), que se perceberam saudáveis e praticavam atividade de lazer fizeram melhor avaliação da QV, assim como as pessoas com menor grau de instrução podem ter preocupações tardias com sua saú$\mathrm{de}^{34}$. Isso traduz a importância da educação em todas as fases da vida, e o grande desafio das gerações futuras para a mudança do perfil do envelhecimento, no sentido de diminuir as desigualdades e favorecer a instrução como forma de melhorar a QV da população que envelhece.

Quanto à variável "Sexo", não houve diferença significativa na QV e na independência funcional de homens e mulheres. No entanto, na literatura observam-se resultados discordantes. Um estudo envolvendo uma amostra representativa da população idosa de Porto Alegre, cujo objetivo foi avaliar a capacidade funcional e identificar sua associação com a QV, demonstrou que homens e mulheres apresentaram diferenças significativas para esses dois construtos, sendo estas mais favoráveis ao sexo masculino ${ }^{35}$. Do mesmo modo, uma pesquisa entre idosos residentes em um município do Sudeste do Brasil observou que o sexo feminino possui percepção mais negativa da QV quando comparado ao sexo masculino ${ }^{36}$.

O domínio do WHOQOL-OLD que obteve escore mais baixo foi "Intimidade", indicando que a QV está mais afetada nesse domínio em relação aos demais. $\mathrm{O}$ domínio envolve questões relacionadas à capacidade de ter relacionamentos íntimos e pessoais. Esse é um tema pouco referido pelos idosos. A sexualidade é uma dimensão inerente a cada pessoa e se manifesta no desejo de contato, intimidade como expressão emocional, prazer, amor e carinho; no entanto, na terceira idade, é vista de forma estereotipada ou mesmo despercebida ${ }^{37}$. Esse mesmo resultado foi encontrado em outro estudo com idosos participantes de grupo de convivência. Os autores consideraram que o fato de a amostra ser constituída na maior parte por mulheres pode justificar esse resultado. As mulheres têm maior probabilidade de enviuvar em idade menos avançada, e têm muito mais anos pela frente ${ }^{38}$.

Não foram encontrados estudos que correlacionassem QV e independência funcional entre idosos participantes de grupos de convivência. No entanto, um estudo que comparou a QV (utilizando questionário Perfil de Saúde de Nottingham) e a independência funcional (utilizando a MIF) entre idosos ativos e sedentários encontrou resultados mais favoráveis ao grupo de idosos ativos, destacando a manutenção da independência funcional associada à $\mathrm{QV}^{39}$.

Os resultados deste estudo reforçam a ideia de que a funcionalidade está diretamente ligada à QV. Essa constatação deve ser considerada na construção de estratégias de prevenção como forma de assegurar, o quanto possível, a independência e a autonomia do idoso.

Em conclusão, a frequência e a participação ativa desses idosos nesse grupo de convivência aumentaram seu repertório de habilidades sociais e sua rede de apoio social e contribuíram para minimizar os sentimentos de isolamento e abandono, fazendo surgir novas amizades e novas oportunidades de aprendizado. Esses espaços devem ser incentivados e mais explorados como forma de promover um envelhecimento bem-sucedido, diminuindo as demandas por serviços de saúde e melhorando a QV dessa população. 


\section{REFERÊNCIAS}

1. Carvalho JAM, Rodríguez-Wong LL. A transição da estrutura etária da população brasileira na primeira metade do século XXI. Cad Saúde Pública. 2008;24(3):597-605. http://dx.doi.org/10.1590/S0102-311X2008000300013

2. Paschoal SMP. Qualidade de vida na velhice. In: Freitas EV, Py L, Nér AL, Cançado FAX, Gorzoni ML, Rocha SM (Eds.). Tratado de geriatria e gerontologia. Rio de Janeiro: Guanabara Koogan; 2002; p.79-84.

3. Moreira MIC, Bedran PM, Carellos SMSD. A família contemporânea brasileira em contexto de fragilidade social e os novos direitos das crianças: desafios éticos. Psicol Rev. 2011;17(1):161-80.

4. Wirth MN. As novas configurações da família contemporânea e o discurso religioso. Seminário Internacional Fazendo Gênero 10 Anais Eletrônicos. Florianópolis; 2013.

5. Nasri F. O envelhecimento populacional no Brasil. Einstein (São Paulo). 2008;6(Supl. 1):S4-6.

6. Fleck MPA (Org.). A avaliação de qualidade de vida: guia para professionais da saúde. Porto Alegre: Artmed; 2008.

7. Young Y, Fan MY, Hebel JR, Boult C. Concurrrent validity of administering the functional independence measure (FIM) instrument by interview. Am J Phys Med Rehabil. 2009;88(9):766-70. http://dx.doi.org/10.1097/PHM.0b013e3181a9f1d6

8. Virués-Ortega J, de Pedro-Cuesta J, Seijo-Martínez M, Saz P, Sánchez-Sánchez F, Rojo-Pérez F, et al. Prevalence of disability in a composite $\geq 75 y$ year old population in Spain: a screening survey based on the International Classification of Functioning. BMC Public Health. 2011;11:176.

http://dx.doi.org/10.1186/1471-2458-11-176

9. Ottenbacher KJ, Hsu Y, Granger CV, Fiedler RC. The reliability of the functional independence measure: a quantitative review. Arch Phys Med Rehabil. 1996;77(12): 1226-32. http://dx.doi.org/10.1016/S0003-9993(96)90184-7

10. Riberto M, Miyazaki MH, Jorge Filho D, Sakamoto H, Battistella LR. Reprodutibilidade da versão brasileira da Medida de Independência Funcional. Acta Fisiátrica. 2001;8(1):45-52.

11. Kawasaki K, Cruz KCT, Diogo MJD. A utilização da Medida de Independência Funcional (MIF) em idosos: uma revisão bibliográfica. Med Reabil. 2004;23(3):57-60.

12. Stevens JP. Applied Multivariate Statistics for the Social Sciences. $2^{\text {nd }}$ ed. New Jersey: Lawrence Erlbaum Associates; 1992; p. 123-7.

13. Hulley SB, Cummings SR, Browner WS, Grady DG, Newman TB. Designing clinical research. Baltimore: Williams \& Wilkins; 1998; p. 218.

14. The World Health Organization Quality of Life Assessment (WHOQOL): development and general psychometric properties. Soc Sci Med. 1998;46(12):1569-85

15. Fleck MP, Chachamovich E, Trentini C. Development and validation of the Portuguese version of The WHOQOL-OLD module. Rev Saúde Pública. 2006;40(5):785-91. http://dx.doi.org/10.1590/S0034-89102006000600007

16. Ajzen I, Fishbein M. Understanding attitudes and predicting social behavior. New Jersey: Prentice Hall; 1980; p.278.

17. Pilger $\mathrm{C}$, Menon MH, Mathias TAF. Características sociodemográficas e de saúde de idosos: contribuições para os serviços de saúde. Rev Latino-Am Enfermagem. 2011;19(5):1230-8. http://dx.doi.org/10.1590/S0104-11692011000500022
18. Almeida AV. O processo de feminização da velhice no município de Viçosa, MG: características, relações e risco social. Dissertação (Mestrado) - Universidade Federal de Viçosa. Viçosa: 2015.

19. Nascimento MR. Feminização do envelhecimento populacional expectativas e realidades de mulheres idosas quanto ao suporte familiar. In: Wong LR. O envelhecimento da população brasileira e o aumento da longevidade: subsídios para políticas orientadas ao bem-estar do idoso. Belo Horizonte: UFMG/Cedeplar, ABEP; 2001.

20. Instituto Brasileiro de Geografia e Estatística (IBGE). Pesquisa nacional por amostra de domicílios: síntese de indicadores 2012. Disponível em: http://www.ibge.gov.br/home/estatistica/ populacao/trabalhoerendimento/pnad2012/default_sintese.shtm. Acesso em: 02 out. 2015

21. Mendes MRSSB, Gusmão JL, Faro ACM, Leite RCBO. A situação social do idoso no Brasil: uma breve consideração. Acta Paul Enferm. 2005;18(4):422-6.

http://dx.doi.org/10.1590/S0103-21002005000400011

22. Lebrão ML, Laurenti R. Saúde, bem-estar e envelhecimento: o estudo SABE no município de São Paulo. Rev Bras Epidemiol. 2005;8(2):127-41.

http://dx.doi.org/10.1590/S1415-790X2005000200005

23. Montilla DER. Informação epidemiológica e populacional: disponibilidade, qualidade e acesso aos sistemas de informação em saúde. Disponível em: http://www5.ensp.fiocruz.br/biblioteca/ dados/txt_549699727.pdf. Acesso em: 02 out. 2015.

24. Miranda LC, Banhato EFC. Qualidade de vida na terceira idade: a influência da participação em grupos. Psicol Pesquisa UFJF. 2008;2(1):69-80

25. Dagios P, Vasconcellos C, Evangelista DHR. Avaliação da qualidade de vida: comparação entre idosos não institucionalizados participantes de um Centro de Convivência e idosos institucionalizados em JI-Paraná/RO. Estud Interdiscip Envelhec. 2015;20(2):469-84.

26. Neri AL. Velhice e qualidade de vida na mulher. In: Neri AL. Desenvolvimento e envelhecimento: perspectivas biológicas, psicológicas e sociológicas. Campinas: Papirus; 2001; p. 161-200.

27. Ferretti F, Beskow GCT, Slaviero RC, Ribeiro CG. Análise da qualidade de vida em idosos praticantes e não praticantes de exercício físico regular. Estud Interdiscip Envelhec. 2015;20(3):729-43.

28. Ferraresi JR, Prata MG, Scheicher ME. Avaliação do equilíbrio e do nível de independência funcional de idosos da comunidade. Rev Bras Geriatr Gerontol. 2015;18(3):499-506. http://dx.doi.org/10.1590/1809-9823.2015.14051

29. Pereira FM, Besse M. Fatores associados à independência funcional de idosos residentes em instituição de longa permanência. Acta Fisiátrica. 2011;18(2):66-70.

30. Pereira KCR, Alvarez AM, Traebert JL. Contribuição das condições sociodemográficas para a percepção da qualidade de vida em idosos. Rev Bras Geriatr Gerontol. 2011;14(1):85-95.

31. Maués CR, Paschoal SMP, Jaluul O, França CC, Jacob Filho W. Avaliação da qualidade de vida: comparação entre idosos jovens e muito idosos. Rev Bras Clin Med. 2010;8(5):405-10.

32. García EL, Banegas JR, Pérez-Regadera AG, Cabrera RH, Rodríguez-Artalejo F. Social network and health-related quality of life in older adults: a population-based study in Spain. Qual Life Res. 2005;14(2):511-20 
33. Faller JW, Melo WA, Versa GLGS, Marcon SS. Qualidade de vida de idosos cadastrados na Estratégia Saúde da Família de Foz do Iguaçu-PR. Esc Anna Nery Rev Enferm. 2010; 14(4):803-10.

http://dx.doi.org/10.1590/S1414-81452010000400021

34. Vitorino LM, Paskulin LMG, Vianna LAC. Qualidade de vida de idosos da comunidade e de instituições de longa permanência: estudo comparativo. Rev Latino-Am Enfermagem. 2013;21(spe):3-11.

http://dx.doi.org/10.1590/S0104-11692013000700002

35. Caneppele MCGL. Capacidade funcional e qualidade de vida de indivíduos idosos de Porto Alegre. Tese (Doutorado) - Universidade Federal do Rio Grande do Sul. Porto Alegre: 2007.
36. Pereira RJ, Cotta RMM, Franceschini SCC, Ribeiro RCL, Sampaio $\mathrm{RF}$, Priore SE, et al. Contribuição dos domínios físico, social, psicológico e ambiental para a qualidade de vida global de idosos. Rev Psiquiatr Rio Gd Sul. 2006;28(1):27-38. http://dx.doi.org/10.1590/S0101-81082006000100005

37. Vieira KFL, Miranda RS, Coutinho MPL. Sexualidade na velhice: um estudo de representações sociais. Psicol Saber Soc. 2012;1(1):120-8.

38. Serbim AK, Figueiredo AEPL. Qualidade de vida de idosos em um grupo de convivência. Sci Med. 2011;21(4):166-72.

39. Gomes Neto M, Castro MF. Estudo comparativo da independência funcional e qualidade de vida entre idosos ativos e sedentários. Rev Bras Med Esporte. 2012;18(4):234-7. http://dx.doi.org/10.1590/S1517-86922012000400003 